\title{
Tabularia
}

TABULARIA Sources écrites des mondes normands médiévaux Les « Précieux Sangs » : reliques et dévotions | 2008

\section{Du culte des reliques à celui du Précieux Sang}

From the Worship of Relics to the Cult of Precious Blood

\section{André Vauchez}

\section{OpenEdition}

\section{Journals}

Édition électronique

URL : http://journals.openedition.org/tabularia/432

DOI : 10.4000/tabularia.432

ISSN : $1630-7364$

Éditeur :

CRAHAM - Centre Michel de Boüard, Presses universitaires de Caen

\section{Référence électronique}

André Vauchez, «Du culte des reliques à celui du Précieux Sang », Tabularia [En ligne], Les " Précieux

Sangs » : reliques et dévotions, mis en ligne le 30 décembre 2008, consulté le 19 avril 2019. URL

http://journals.openedition.org/tabularia/432 ; DOI : 10.4000/tabularia.432 


\title{
Du culte des reliques à celui du Précieux Sang From the Worship of Relics to the Cult of Precious Blood
}

\author{
André VAUCHEZ \\ Académie des inscriptions et belles-lettres, Institut de France \\ avauchez@wanadoo.fr
}

Résumé:

Le culte des reliques des saints a joué un rôle majeur dans la christianisation de l'Europe pendant le Haut Moyen Âge. À partir du XI siècle, la religiosité des fidèles a été marquée par une dévotion croissante envers le Christ et les Apôtres, qui se traduisit par une recherche de tous les signes tangibles de leur vie terrestre. C'est dans ce contexte de pèlerinages et de croisades en Terre Sainte que se situent les miracles attribués au Saint-Sang ou au Précieux Sang de Jésus, avant que ces derniers ne deviennent, à partir du XIII siècle, des preuves de la présence réelle du Fils de Dieu dans le sacrement de l'eucharistie.

Mots clés: reliques, reliquaire, saints, sang, miracle, pèlerinage, Orient, Terre Sainte, Jérusalem.

Abstract:

The worship of saints'relics played a major role in the Christianisation of Europe in the High Middle Ages. From the 11th century onwards, the religious fervour of the faithful was marked by a growing devotion to Christ and the Apostles, which found expression in a quest for any material sign of their earthly existence. The miracles attributed to the Holy Blood or the Precious Blood of Jesus first arose in the context of pilgrimages and crusades to the Holy Land, before becoming evidence of the actual presence of the Son of God in the sacrament of the Eucharist.

Keywords: relics, reliquary, saints, blood, miracle, pilgrimage, East, Holy Land, Jerusalem.

Alors que l'étude historique des formes de la sainteté, des textes hagiographiques et des miracles a connu un essor remarquable chez les médiévistes dès le milieu des années 1960, les reliques ne sont entrées qu'assez récemment dans le champ de la recherche. En effet, si l'on met de côté les ouvrages pionniers d'André Grabar ${ }^{1}$, axés sur l'histoire de l'architecture religieuse de l'Antiquité tardive, et de A. Frolow ${ }^{2}$, qui concerne surtout le monde byzantin, il a fallu attendre la fin des années 1970, avec les livres de Patrick Geary sur les vols de

1. GrabAR, 1946.

2. Frolow, 1961.

Tabularia "Études», n 8, 2008, p. 81-88, 30 décembre 2008 http://www.unicaen.fr/mrsh/craham/revue/tabularia/print.php?dossier=dossier8\&file=01vauchez.xml 
reliques ${ }^{3}$ et de Martin Heinzelmann sur les récits de translation ${ }^{4}$ pour que l'importance de ces «fragments d'éternité» présents au cœur des sociétés médiévales et modernes commence à être bien perçue ${ }^{5}$. Encore les grandes synthèses où la centralité des reliques dans l'univers mental et la vie des hommes de ce temps est nettement affirmée sont-elles assez récentes, qu'il s'agisse de l'ouvrage d'Arnold Angenendt ${ }^{6}$, des actes du colloque de Boulogne-sur-mer édités par Edina Bozoky et Anne-Marie Helvetius ${ }^{7}$, ou encore du vaste panorama brossé par Henk Van $\mathrm{Os}^{8}$. Sous l'heureuse influence de ces auteurs et de quelques autres dont l'énumération risquerait de transformer cette introduction en un palmarès fastidieux, une prise de conscience s'est opérée au niveau européen parmi les chercheurs et les travaux relatifs aux reliques se sont multipliés aussi bien dans le domaine de l'histoire religieuse que dans celui de l'histoire de l'art, tant il est vrai que, comme l'a noté Marie-Madeleine Gauthier, «à la charge apotropaïque et thaumaturgique des reliques, le reliquaire ajoutait la vertu doctrinale des images" ${ }^{9}$. Ces objets sacrés, "parcelles de l'au-delà offertes à la vénération des vivants ${ }^{10}$, furent en effet vénérés mais aussi manipulés et mobilisés par les divers pouvoirs - gouvernants, institutions ecclésiastiques ou entités politiques - qui s'en servirent comme marqueurs de leur autorité ou de leur territoire et ils sont maintenant devenus un sujet d'étude pour les historiens du politique ${ }^{11}$.

L'importance attribuée aux reliques du Christ, de la Vierge et des saints constitue en effet un des traits fondamentaux de la civilisation médiévale. C'est sur les reliques ou les évangiles que se prêtaient les serments les plus solennels, en particulier quand un grand de ce monde devait se justifier face à des accusations d'une extrême gravité, et c'étaient elles qu'on portait en procession à travers la ville à l'approche de l'ennemi ou d'une épidémie. En outre, il faut mentionner le fait que, sans les offrandes recueillies auprès des fidèles à l'occasion des ostensions de reliques ou à l'occasion de leurs pèlerinages aux tombeaux des saints, les grands sanctuaires de la chrétienté et les cathédrales, de Saint-Martin de Tours à Saint-Jacques de Compostelle, n'auraient pas pu être construits ou reconstruits de façon aussi somptueuse. On peut donc dire sans exagération que, jusqu'au XII ${ }^{e}$ siècle, le culte des saints s'est dans une large mesure identifié à celui des reliques.

Comment en est-on arrivé là? C'est ce que je voudrais essayer de retracer dans ces quelques pages qui ne prétendent pas à l'originalité, mais où je chercherai à situer le pouvoir communément attribué aux reliques dans le contexte religieux de l'époque. La croyance dans la vertu des reliques remonte aux premiers

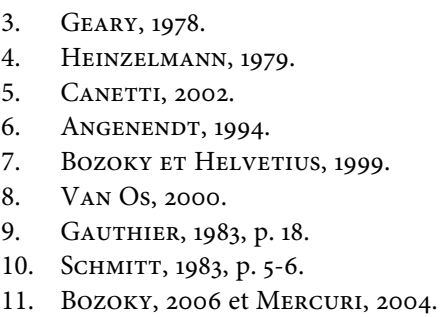


siècles du christianisme et s'est développée parallèlement au culte des martyrs. Aux yeux des premiers chrétiens en effet, l'exemple donné par ces derniers, qui avaient persévéré dans la foi au Christ jusqu'au sacrifice suprême en dépit des supplices qui leur étaient infligés, attestait la présence en eux d'un élément divin. Leurs corps, devenus temples du Saint-Esprit, ne pouvaient pas ne pas conserver après leur mort la trace du pouvoir surnaturel qui les avait animés jusqu'au bout et qui les avait rendus semblables au Christ. Ce fut ainsi que, à partir du III siècle, on prit l'habitude de rendre des honneurs particuliers aux restes des martyrs, qu'il s'agisse du corps entier, de la tête ou de simples draps tachés par leur sang. À partir du moment où l'empire romain cessa de persécuter les chrétiens, on vit s'affirmer d'autres types de saints comme les confesseurs (saint Athanase ou saint Hilaire par exemple) qui, bien que n'ayant pas subi le martyre sanglant, s'étaient prodigués avec courage pour la propagation et la défense de la foi chrétienne contre ses adversaires, ou les ascètes qui s'étaient signalés par l'intensité de leurs mortifications et de leur combat spirituel, comme saint Antoine et les premiers moines des déserts d'Egypte et de Syrie. Quand le culte chrétien fut officiellement reconnu, au cours du IVe siècle, des édifices commémoratifs (memoriae, martyria) furent construits en leur honneur sur le lieu ou à proximité de leur sépulture, auxquels succédèrent, dans un certain nombre d'endroits, de grandes basiliques destinées à protéger les reliques et à stimuler la dévotion des fidèles à leur égard. Les tombes des saints et les églises qui leur étaient dédiées constituaient autant de points de contact privilégiés entre le Ciel et la terre, et les villes qui avaient l'avantage de posséder de telles reliques en tiraient un grand profit. Ainsi, c'est à cette époque que les papes commencèrent à fonder leur revendication d'une primauté de l'Église romaine par rapport aux autres Églises sur le fait que la Ville éternelle possédait les corps des apôtres Pierre et Paul. À quoi le clergé de Constantinople et le pouvoir impérial répliquèrent en concentrant dans la nouvelle capitale de l'empire des reliques des saints André, Luc, Timothée, Phocas et du prophète Samuel, dont les restes furent conduits en procession solennelle de Jérusalem aux rives du Bosphore. Plus généralement, toute cité du monde romain, tant en Orient qu'en Occident, qui avait la chance de posséder le corps d'un ou plusieurs saints, se considérait comme purifiée par «le sang immolé des martyrs qui a chassé la race ennemie des démons», selon les paroles du poète Prudence au début du $\mathrm{V}^{\mathrm{e}}$ siècle. Et plus on pouvait accumuler de reliques en un lieu donné, plus on se sentait protégé de toute espèce de mal comme par une sorte de bouclier spirituel dont l'efficacité ne pouvait être mise en doute.

Un autre phénomène se conjugua au précédent pour donner au culte des reliques une place centrale - qu'il n'avait pas initialement - dans la nouvelle religion. Son importance s'accrut en effet de façon considérable à partir du moment où le christianisme gagna les campagnes et surtout où il fut adopté par des populations germaniques étrangères aux traditions et aux valeurs culturelles de la civilisation romaine. Incapable d'accéder à la compréhension des dogmes et du discours théologique, les "païens» et les barbares privilégièrent au sein du christianisme les manifestations les plus sensibles de leur nouveau Dieu et de ses amis, 
les saints, dont ils expérimentaient le pouvoir à travers les victoires militaires et les miracles de ses adeptes, et adoptèrent avec enthousiasme le culte des reliques, considérées par eux comme des sortes d'amulettes ou de talismans. La virtus le pouvoir surnaturel qui émanait des reliques - leur apparaissait comme une force immanente dont ils constataient l'efficacité sans nécessairement en comprendre clairement l'origine; le rapport avec la foi chrétienne était assuré par le clergé, qui était seul en mesure d'établir une relation de cause à effet entre les miracles qui se produisaient sur les tombes des saints et les mérites réels ou supposés de leurs auteurs, attestés par un texte écrit - Passion ou Vita -. Saint Augustin aimait rappeler que "les saints méritent l'honneur du culte uniquement dans la mesure où ils ont été les serviteurs du Seigneur et ont été glorifiés par lui» et saint Maxime, le premier évêque de Turin au début du Ve siècle, affirmait que «la sainteté des reliques ne peut sauver que ceux qui imitent les saints». Il n'est pas sûr pour autant que ces considérations aient bien été présentes à l'esprit des fidèles qui vénéraient leurs reliques et cherchaient à établir par leur intermédiaire une communication bénéfique avec l'au-delà.

L'Église fut bien obligée de tenir compte de cette situation nouvelle et d'adapter sa pastorale à la pression insistante des masses. Comme l'a bien montré Peter Brown dans un ouvrage devenu classique, les évêques eux-mêmes ne tardèrent pas à se lancer à la recherche de nouvelles reliques, surtout dans les régions qui en étaient dépourvues, et à devenir les protagonistes de leur culte ${ }^{12}$. C'est ainsi qu'on assista, à partir de la fin du IV ${ }^{e}$ siècle, à la multiplication des «inventions" (du latin invenire, trouver) de corps saints, comme celle qui se produisirent à Milan dont l'évêque Ambroise découvrit successivement les restes des saints martyrs Gervais et Protais (388) et Agricola et Vital (393). Ce phénomène s'amplifia avec l'exhumation, en 415, près de Jérusalem, des reliques de saint Etienne et de celle de saint Barnabé à Chypre en 458. Grégoire de Tours raconte qu'en Gaule, au début du VIe siècle, l'évêque de Langres fit ouvrir un beau sarcophage antique où reposaient les restes d'un personnage inconnu envers lequel la population de Dijon manifestait une grande vénération. Le prélat décida qu'il s'agissait des reliques de saint Bénigne, martyr d'origine grecque dont on ne savait pas grand chose mais dont une Vie, rédigée quelques années plus tard, souligna opportunément qu'il avait joué un rôle important dans l'évangélisation de la Bourgogne. C'est au-dessus de cette tombe, où se produisirent de nombreux miracles, que devait se développer, à l'époque carolingienne, la grande abbaye de SaintBénigne qui fut réformée au XI ${ }^{e}$ siècle par Guillaume de Volpiano. En dernière analyse, on a l'impression que ces «inventions» de reliques, qui furent fréquentes tout au long du Moyen Âge, ont souvent correspondu à des tentatives de la hiérarchie ecclésiastique visant à reprendre en main la dévotion populaire et à l'orienter vers des figures orthodoxes, sinon toujours authentiques.

La recherche effrénée des reliques de la part des communautés chrétiennes devait fatalement conduire à la fragmentation et à la dispersion des corps saints.

12. BROWN, 1984. 
S'appuyant sur les affirmations de théologiens comme Grégoire de Nazianze selon lesquels la puissance des martyrs résidait autant dans le moindre fragment de leurs restes que dans leur corps tout entier, les clercs orientaux furent les premiers à démembrer les corps des serviteurs de Dieu, de façon à en faire profiter le plus grand nombre possible d'églises, et ceci malgré une disposition du code théodosien (438) qui interdisait de déplacer les corps des défunts ou de violer leur intégrité. En Occident, on respecta plus longtemps les prescriptions du droit romain dans ce domaine et, en 594, le pape Grégoire le Grand, en réponse à une demande de l'impératrice Constantine qui lui demandait d'envoyer de Rome à Constantinople la tête de saint Paul, affirma qu'il n'était pas dans les habitudes du peuple romain de toucher aux cadavres, même pour en prélever une parcelle. En guise de consolation et pour atténuer sans doute la brutalité de son refus, il lui fit parvenir des brandea, c'est-à-dire des morceaux de tissus qui avaient été déposés sur la tombe du saint et s'étaient imprégnés de sa virtus. Mais le respect des anciennes normes ne survécut pas aux attaques lancées par les Lombards contre Rome qui conduisirent les papes des VII et VIII ${ }^{e}$ siècles à faire exhumer de nombreux corps qui reposaient en dehors des murs de la Ville pour les transporter à l'intérieur de la cité, où ils furent ensuite divisés en fragments qui allèrent enrichir les autels et les trésors des églises dans les régions de la chrétienté qui en étaient dépourvues. À partir du IX ${ }^{e}$ siècle, on vit affluer à Rome quantité d'évêques et d'abbés francs qui, par l'achat ou le vol, se procuraient de précieux restes, en particulier dans les Catacombes, pour les emporter en France ou en Allemagne. Ces pillages systématiques, à l'occasion desquels furent mises en circulation quantité de fausses reliques, ne devaient plus s'interrompre avant le $\mathrm{XI}^{\mathrm{e}}$ siècle et furent même accrus par l'évolution des usages liturgiques, puisque l'habitude se prit alors en Occident d'insérer des reliques dans tous les autels où était offert le sacrifice eucharistique. Ainsi, même la plus modeste église de village se devait d'en posséder, ce qui contribua à accélérer le processus de démembrement et de dispersion des corps saints.

$\mathrm{Au}$ terme de cette évolution, autour de l'an Mille, les reliques étaient partout présentes et occupaient une place centrale dans la vie religieuse des fidèles, comme le montrent bien les écrits d'un Raoul Glaber. L'Église ne pouvait que s'en réjouir dans la mesure où leur diffusion avait constitué un puissant instrument de christianisation pour la société occidentale. En particulier, le culte des reliques contribua à diffuser chez les laïcs un sens très fort et concret de la communion des saints, c'est-à-dire de cette mystérieuse communication entre les vivants et les morts en vertu de laquelle les hommes et les femmes d'ici bas pouvaient bénéficier des mérites des saints du paradis, dont l'intercession pouvait les aider - ou aider leurs proches et leurs défunts - à affronter les difficultés de l'existence ou les dangers de l'au-delà. Mais, à partir du XII ${ }^{e}$ siècle, des voix commencèrent à s'élever, au sein du monachisme, pour dénoncer les périls que cette réification du sacré faisait courir à la vraie foi. Vers 1100, Guibert de Nogent écrivit un traité De pignoribus sanctorum (sur les reliques des saints) dans lequel il dénonça certains abus qui accompagnaient cette dévotion: constatant que dans la France de son temps, on ne vénérait pas moins de trois têtes de saint Jean Baptiste en trois 
endroits différents, il en vint à se demander s'il ne valait pas mieux laisser les saints jouir du repos éternel, que du reste ils avaient bien mérité, plutôt que de rendre des honneurs indus à des objets douteux. Dans l'immédiat, ces critiques n'entamèrent en rien la foi des clercs et des fidèles dans la vertu de leurs reliques, mais elles furent à l'origine d'une réflexion qui amena l'Église romaine, à partir de la seconde moitié du XII siècle, à mettre l'accent, à travers l'hagiographie et bientôt les procès de canonisation, sur l'exemple fourni par les saints de leur vivant et sur leurs vertus plutôt que sur le rayonnement bénéfique qui émanait de leurs restes ${ }^{13}$.

Mais, dès le $\mathrm{XI}^{e}$ siècle, le culte des reliques avait commencé à évoluer de l'intérieur sous l'influence d'un nouveau courant de spiritualité, qui préconisait le retour à la Vie Apostolique, c'est-à-dire à l'idéal offert par la première communauté chrétienne de Jérusalem, réunie autour des Apôtres, telle qu'elle est décrite dans les Actes des Apôtres (IV, 32-35) et, plus largement au modèle de l'Église primitive (Ecclesiae primitivae forma). Sous l'effet d'une pastorale carolingienne et post-carolingienne que nous connaissons mal mais dont nous percevons les effets et les manifestations, la piété des clercs et des fidèles se recentra sur le Christ, la Vierge Marie - à laquelle on commença à dédicacer presque toutes les cathédrales -, saint Jean Baptiste et les Apôtres. Cela se traduisit par un intérêt accru pour les lieux où ces derniers avaient vécu, ce qui favorisa l'essor du pèlerinage à Jérusalem et en Terre Sainte où les fidèles commencèrent à se rendre en grand nombre à la suite de leurs évêques bien avant qu'il soit question de croisade, mais aussi un afflux croissant de pèlerins à Saint-Jacques de Compostelle après la reconstruction de la basilique qui avait été détruite par les Sarrasins peu avant l'an Mille. Parallèlement, on voit s'intensifier le mouvement de transfert des reliques de nombreux saints prestigieux d'Orient vers l'Occident, inauguré en 847 par la capture des reliques de saint Marc par les Vénitiens à Alexandrie et qui devait se poursuivre et s'intensifier jusqu'au pillage systématique des églises de Constantinople par les «Francs» lors de la $4^{e}$ croisade, en 1204. À travers tous ces furta sacra s'exprime l'intérêt croissant éprouvé par les Latins pour toutes les traces concrètes de la présence du Christ et de ses compagnons: en 1095, lors du siège d'Antioche, les croisés découvrent miraculeusement la Sainte Lance qui avait percé le côté du Sauveur sur le Golgotha; un peu partout en Occident - à la cathédrale de Chartres puis à celle de Prato, en Toscane -, apparaissent des ceintures de la Vierge qui font l'objet d'une intense dévotion de la part des fidèles; au XII siècle, on commence à vénérer à Cadouin, en Dordogne, le SaintSuaire du Christ (en fait, un faux des années 1100, peint sur un tissu égyptien où étaient tissées des formules de bénédiction en arabe adressées par Mahomet...) qui aurait été ramené en France par l'évêque Adhémar du Puy, tandis que le culte du Volto Santo attirait à Lucques des foules venues y contempler la face tragique du Crucifié. C'est dans ce contexte qu'il faut situer l'apparition de la dévotion au Saint-Sang ou Précieux Sang du Sauveur, à Fécamp, vers $990^{14}$, puis à

13. VAUCHEZ, 1988.

14. Le MAHO, 2004, p. 93-106. 
Mantoue (Italie du Nord), dans une manifestation spectaculaire en 1049, puis par le Saint-Sang de Bruges au XII ${ }^{\mathrm{e}}$ siècle. À Venise, au début du XIII ${ }^{\mathrm{e}}$ siècle, on voit entrer dans le trésor de Saint-Marc un reliquaire-ostensoir du sang miraculeux, conservé dans un flacon de verre d'époque fatimide envoyé de Constantinople par le doge Dandolo, qui contient une fibre de coton teintée du sang de Jésus et portant l'inscription suivante: «Tu me possèdes, moi le Christ, toi qui portes le sang de ma chair ${ }^{15}$. Il se serait agi de gouttes de sang distillées par la Vraie Croix, lorsque celle-ci fut retrouvée à Jérusalem par sainte Hélène, après que les Juifs l'eurent profanée à coups de couteau, selon le récit traditionnel. Au fur et à mesure qu'on avance dans le temps, les traces sanglantes vénérées ici où là dans la chrétienté furent présentées comme des gouttes de sang recueillies par les Apôtres après l'agonie du Christ à Gethsemani, ou par Joseph d'Arimathie après la descente de croix et lors de la mise au tombeau, dont certaines seraient même tombées miraculeusement dans un calice, ce qui fut à l'origine du thème et du cycle littéraire du Saint Graal. Mais toutes ces dévotions seront relancées et amplifiées, à partir du XIII ${ }^{\mathrm{e}}$ siècle, par l'accent mis par l'Église sur la présence réelle dans les espèces consacrées lors du sacrifice de la messe, dans le cadre de sa réaction contre la contestation des sacrements par les hérétiques ${ }^{16}$. Dès lors, le culte du Précieux Sang changera de registre et se traduira par des miracles eucharistiques, comme ceux de Bolsena au XIII siècle ou Wilsnack au XVe, où l'élément central n'était plus la personne même du Sauveur mais l'identité physique, au sens le plus réaliste du terme, du pain et du vin consacrés au corps et au sang du Christ.

\section{Bibliographie}

Angenendt, Arnold, Heilige und Reliquien: die Geschichte ihres Kultes vom frühen Christentum bis zur Gegenwart, München, C. H. Beck, 1994, 470 p.

Bozoky, Édina, La politique des reliques de Constantin à saint Louis, Paris, Beauchesne, 2006, $315 \mathrm{p}$.

Bozoky, Édina et Helvétıus, Marie, Les Reliques: objets, cultes, symboles, Actes du Colloque international de l'Université du Littoral-Côte d'Opale (Boulogne-surmer), 4-6 septembre 1997, Turnhout, Brepols, 1999, 336 p.

Brown, Peter, Le culte des saints. Son essor et sa fonction dans la chrétienté latine, Paris, Éditions du Cerf, 1984, $164 \mathrm{p}$.

Bynum, Caroline, Wonderful Blood. Theology and Practice in Late Medieval Germany and Beyond, Philadelphia, University of Pennsylvania Press (coll. The Middle Ages series), 2007, XVIII-402 p.

CANetti, Luigi, Frammenti d'eternità. Corpi e reliquie tra Antichità e Medioevo, Rome, Viella, 2002, $238 \mathrm{p}$.

Frolow, Anatole, La relique de la vraie croix. Recherches sur le développement d'un culte, Paris, Institut français d'études byzantines, 1961, 695 p.

15. GAUTHIER, 1983.

16. Sur cette évolution, cf. BynUM, 2007 et VinCENT, 2001. 
Gauthier, Marie-Madeleine, Les routes de la foi. Reliques et reliquaires de Jérusalem à Compostelle, Fribourg, Office du Livre, 1983, 220 p.

Geary, Patrick J., Furta sacra. Thefts of Relics in the Central Middle Ages, Princeton, Princeton University Press, 1978, XVI-227 p.

Grabar, André, Martyrium. Architecture: recherches sur le culte des reliques et l'art chrétien antique, Paris, Collège de France, 1946, 3 vol.

Heinzelmann, Martin, Translationsberichte und andere Quellen des Reliquienkultes, Turnhout, Brepols (Typologie des sources du Moyen Âge occidental), 1979, $125 \mathrm{p}$.

Mercuri, Chiara, Corona di Cristo, corona di re. La monarchia francese e la corona di spine nel Medioevo, Rome, Edizioni di Storia e Letteratura (coll. Centro alti studi in scienze religiose), 2004, X-246 p.

Schmitт, Jean-Claude (dir.), Les saints et les stars, Paris, Beauchesne, 1983, 302 p.

Van Os, Henk, The Way to Heaven. Relics Veneration in the Middle Ages, Baarn, de Prom, 2000, $223 \mathrm{p}$.

Vauchez, André, La sainteté en Occident aux derniers siècles du Moyen Âge d'après les procès de canonisation et les documents hagiographiques, Rome-Paris, École française de Rome (coll. Bibliothèque des Écoles françaises d'Athènes et de Rome), $2^{\mathrm{e}}$ éd., 1988, X-771 p.

Vincent, Nicholas, The Holy Blood. King Henry III and the Westminster Blood Relic, Cambridge, Cambridge University Press, 2001, XIII-254 p. 